\title{
Erratum to: A numerical investigation of the 1783 landslide-induced catastrophic tsunami in Scilla, Italy
}

Filippo Zaniboni ${ }^{1} \cdot$ Alberto Armigliato $^{1} \cdot$ Stefano Tinti $^{1}$

\section{Erratum to: Nat Hazards (2016) 84:S455-S470 DOI 10.1007/s11069-016-2461-3}

In the initial online publication of this article the family names and given names of all three authors had been reversed. The names should be as displayed in this erratum. The original article has now been corrected as well.

The online version of the original article can be found under doi:10.1007/s11069-016-2461-3.

\section{Filippo Zaniboni}

filippo.zaniboni@unibo.it

1 Dipartimento di Fisica e Astronomia, Alma Mater Studiorum - Università di Bologna, CONISMA, viale Carlo Berti Pichat 8, 40127 Bologna, Italy 